\title{
Soft tactile sensing: retrieving force, torque and contact point information from deformable surfaces
}

\author{
Simone Ciotti* ${ }^{* \dagger}$, Teng Sun ${ }^{\ddagger}$ Edoardo Battaglia ${ }^{\dagger}$, Antonio Bicchi ${ }^{* \dagger \S}$, Hongbin Liu ${ }^{\ddagger}$ and Matteo Bianchi ${ }^{\dagger}$ \\ * Soft Robotics for Human Cooperation and Rehabilitation Research Line, Instituto Italiano di Tecnologia, Via Morego 30, 16163, Genova, Italy \\ ${ }^{\dagger}$ Research Center E. Piaggio, University of Pisa, Largo L. Lazzarino 1, 56126 Pisa, Italy \\ simone.ciotti, e.battaglia, bicchi, matteo.bianchi@centropiaggio.unipi.it \\ \$Haptic Mechatronics and Medical Robotics (HaMMeR) Lab, King's College London, WC2R 2LS, London, UK \\ teng.sun, hongbin.liu@kcl.ac.uk

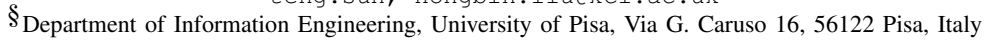

\begin{abstract}
Intrinsic Tactile Sensing (ITS) is a well-established technique, relying on force/torque and geometric surface description to find contact centroids. The method works well for rigid surfaces. However, finding a solution for deformable surfaces is an open issue. This work presents two solutions to extend ITS to deformable surfaces, relying on force-deformation characteristics of the surface under exploration: (i) a closed-form approach that calculates the contact centroid using standard ITS, but on a shrunk geometry approximating the deformed surface; (ii) an iterative procedure that takes into account soft surface deformation, and force/torque equilibrium to minimize a cost function. We have tested both using ellipsoid silicone specimens, with different softness levels and indented along different directions. Both linear and quadratic fitting for the force-indentation behavior were employed. The two methods have distinct advantages and limitations. However, a combination of two methods, using one to produce the initial guess for the other, turns out to be very effective. Indeed, in our validation this solution showed convergence under $1 \mathrm{~ms}$, attaining errors lower than $1 \mathrm{~mm}$. The proposed approaches were implemented in a ROS-based toolbox, integrating both solutions.
\end{abstract}

\section{INTRODUCTION}

A correct characterization of contact represents a fundamental topic in robotics: it is used in classic mathematical framework for robotic grasp [1], [2], [3], for advanced human robot interaction [4], [5], to analyze slippage or friction properties as in [6], [7], [8] and for planning [9]. A possible approach to contact measurement is tactile arrays [4], [10], [11], [12], [13], which build an artificial skin obtaining a direct measurement of contact through sensorized patches. However, this solution typically only identifies the patch over which the contact is happening [14], and can not return six axes measurements.

To obtain a complete description of the contact phenomenon, force/torque components and contact location are needed. Traditionally this is done through the Intrinsic Tactile Sensing (ITS) approach described in [15], which relies on measurements from six axis force/torque sensors to determine the location of the contact centroid. This algorithm takes into account a soft finger contact model for a compressive contact traction distribution on a convex surface. In [16], [17], and [18] implementations of this algorithm are reported, which enable to compute the contact centroid on different rigid ellipsoidal surfaces. However, if the contact occurs on a deformable surface, the deformed shape can become nonconvex, and the algorithm in [15] can no longer be applied
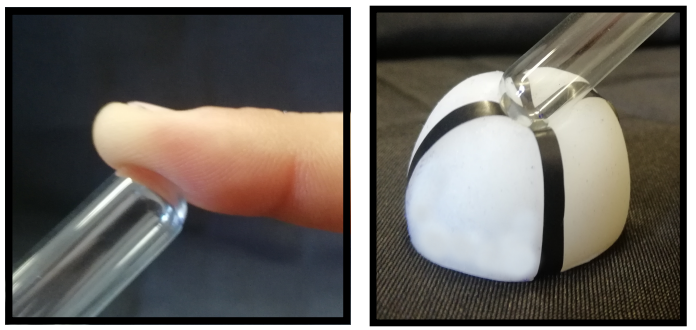

Fig. 1: Soft finger (left) and artificial soft surface (right) in contact with a rigid object.

to compute the contact location. This issue occurs mainly in two cases: when considering deformable sensorized objects, but also when considering the problem of applying ITS to retrieve contact information on soft tactile sensors, as it may be the case of sensorized deformable fingerpads for robotic hands. Figure 1 shows an example of this in a soft finger pad and an artificial soft surface.

Accurate contact location information in deformable finger pads would enable robotic manipulation devices to explore the unknown and unstructured environment more smoothly and adaptively [19], especially when there are unpredicted bumps on the way or sudden changes in surface curvatures [20]. Complete haptic data contain rich information related to the friction coefficients, roughness, surface outline, and other properties of the contact object. Indeed, interpreting this haptic information can facilitate an understanding of the interacted objects with filters or machine learning methods [21]. Under this regard, interesting biomimetic soft sensing solutions were proposed, e.g. to get force, vibration, and temperature information based on impedance changes of the electrodes as in [22] or to retrieve deformation of the sensing surface through optics as in [23]. However, although promising, none of these can provide a precise estimation of contact centroid location. To model contact with soft surfaces, which includes contact location determination, an accurate characterization typically involves Finite Elements Modeling [24], [25]. These approaches however struggle with obtaining a measurement of contacts compatible with real-time constraints, which can be necessary when targeting robotic grasp control.

An attempt to extend the ITS to deformable surfaces in a real time application was done in [26], where a linear characterization of the soft material was used to visually 
reconstruct the deformed shape of a foam plane assembled over a rigid core. However, no quantitative analysis of the error was performed, and only a linear characterization for a planar surface was considered. In [19] the problem of retrieving contact location was applied to the case of a rigid fingertip with a soft layer of skin, under the assumption of single contact and force normal to the surface. From the known surface geometry and mechanical properties of the soft tip, and the measured forces and torques from the 6-axis sensor, the contact equilibrium system equations were derived, leading to contact centroid estimation optimization problem. However, the choice of the initial guess remained a critical point, which strongly influenced the quality of the estimation and lead to issues with convergence when a quadratic approximation was used to model the soft material force-indentation behavior. Furthermore, the case of a completely soft finger was not considered.

Here we propose a characterization-based approach to extend ITS to the general case of ellipsoidal deformable surfaces, where the effect of a contact leads to a non-convex deformed surface. Two different methods were evaluated to estimate the location of the Contact Centroid (CC) on the deformed surface: (i) a closed-form approach that simply calculates the contact centroid location using the standard ITS, but on a shrunk ellipsoid obtained from the material characterization, and (ii) the iterative approach introduced in [19], which was tested with initial guesses defined (ii-a) from the contact centroid as would have happened on a rigid surface, i.e. the initial $\mathrm{CC}$, and (ii-b) assigned with method (i). The performance was evaluated on two silicone specimens with different stiffness, by comparing the estimated $\mathrm{CC}$ with the real $\mathrm{CC}$ as measured by an indenter, with the specimens being probed from different directions. Two types of fitting, linear and quadratic, were used to characterize force-indentation behavior of the materials.

\section{INTRINSIC TACTILE SENSING}

Although the theory behind ITS is relatively complex, in practice it is quite simple to utilize and offers an enormous potential to measure manipulation (see for example[18], [16], and [17|). Since it is close to human finger interaction with external objects, the soft finger contact type is the most general case among the contact models typically used in robotics [27], and it is widely used in practical manipulation [2]. For this reason the ITS assumes a soft finger contact model.

A significant problem simplification can be achieved with the substitution of the unknown distribution of contact tractions with an equivalent force and moment, comprised of a resultant compressive force $\mathbf{p}$, and a resultant moment $\mathbf{q}$ normal to the surface in the contact centroid $\mathbf{c}=\left[c_{x}, c_{y}, c_{z}\right]^{T}$. The $\mathrm{CC}$ has some very useful properties, which make it a desirable point to sense, and can be used for grasp analysis [2]. To implement the ITS, we need a surface $S$ attached to a six axis force/torque sensor. The force/torque sensor measures all three components of both the resultant force $\mathbf{f}=\left[f_{x}, f_{y}, f_{z}\right]^{T}$ and the resultant moment $\mathbf{m}=\left[m_{x}, m_{y}, m_{z}\right]^{T}$ with respect to the reference frame $\{\mathrm{S}\}$.

The sensorized surface can be described by the implicit relation $S(\mathbf{r})=0$ where $\mathbf{r}=\left[r_{x}, r_{y}, r_{z}\right]^{T}$ is a point on the surface defined w.r.t. $\{\mathrm{S}\}$. The surface $S$ should have continuous first derivatives, so that a normal unit vector $\mathbf{n}$ can be defined at every point on $S$. The measurable quantities $\mathbf{f}$ and $\mathbf{m}$ are related to the unknowns $\mathbf{c}, \mathbf{p}$ and $\mathbf{q}$ (i.e. the torque parallel to the unit vector $\mathbf{n}$ evaluated in $\mathbf{c}$ ), by force and moment balance equations,

$$
\begin{aligned}
\mathbf{f} & =\mathbf{p} \\
\mathbf{m} & =\mathbf{q}+\mathbf{c} \times \mathbf{p}
\end{aligned}
$$

that together the relation $S(\mathbf{r})=0$ characterize the contact sensing problem. Due the CC properties, the solution of the contact sensing problem exists and it is unique for a compressive force distribution and a convex surface $S$.

\section{A. ITS for rigid ellipsoidal surfaces}

We will consider in the following only general ellipsoidal surfaces. This choice is motivated by several reasons. First, ellipsoids approximate, up to the second order, any continuous convex surface. Second, very common surfaces, such as spheres, cylinders, and planes, can be regarded as limit cases of an ellipsoid. Finally, the ellipsoid assumption is standard in contact mechanics.

For the ellipsoidal surface family, the contact sensing problem can be written in the form

$$
\begin{aligned}
\Gamma \mathbf{c} & =\mathbf{m} \\
\mathbf{c}^{T} A^{2} \mathbf{c} & =1
\end{aligned}
$$

where $\Gamma$ is a $3 \times 3$ matrix whose elements are functions of the measured force components, and $A$ is a constant coefficient matrix that is function of the surface geometry. A complete and in-depth explained solution of the ITS problem for the ellipsoidal surface family (i.e. ellipse, sphere, cylinder, and plane) can be found in [15].

\section{Intrinsic Tactile Sensing for Soft Surfaces}

In this section, we take into account the ITS problem solution considering deformable ellipsoidal surfaces. When a force is applied on a deformable object, the surface geometry changes and its shape becomes non-convex: in this case the ITS algorithm still provides a solution to the contact sensing problem, but the solution uniqueness is not guaranteed. Using the traditional ITS approach and the general case of an ellipsoid $S$, the coordinates of the contact point $\mathbf{c}$ are constrained to stay on the non-deformed surface, causing an error in the CC computation that is generated by the surface deformation. In this case, the computation error can be reduced with the following approach.

For a deformed surface $S$ we make the assumption that the contact occurs on the surface $S^{\prime}$, as in Fig. $2 . S^{\prime}$ has the same origin of $S$, but its principal axes are scaled w.r.t. the principal axes of $S$. The deformation $\Delta \mathbf{d}=\left[\Delta d_{x}, \Delta d_{y}, \Delta d_{z}\right]^{T}$ of surface $S$ is related to the external force $\mathbf{f}$ through the equation $\mathbf{f}=\mathbf{h}(\Delta \mathbf{d})$, where $\mathbf{h}(\cdot)$ is the deformation estimation function, which is dependent on the material properties: the estimate of the $\mathrm{CC}$ is more accurate when $\mathbf{h}(\cdot)$ offers a good modeling of the surface force-deformation behavior. 


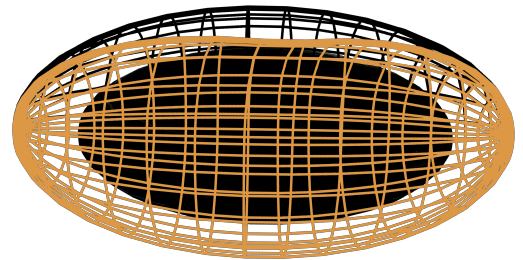

Fig. 2: Non-deformed surface $S$ in black wireframe, deformed surface in orange wireframe and scaled surface $S^{\prime}$ in solid.

In the definition of contact sensing problem for ellipsoidal surfaces reported in [15], the free parameters are the surface principal axes $a, b$, and $c$. Then $S^{\prime}$ can be described throughout the equation

$$
S^{\prime}(\mathbf{c})=\frac{c_{x}^{2}}{\left(a-\Delta d_{x}\right)^{2}}+\frac{c_{y}^{2}}{\left(b-\Delta d_{y}\right)^{2}}+\frac{c_{z}^{2}}{\left(c-\Delta d_{z}\right)^{2}}-1=0
$$

and the CC can be computed exactly on $S^{\prime}$.

\section{A. Closed-form Method}

In the closed-form method, which we defined in the Introduction as (i-a), the unknowns $\mathbf{p}, \mathbf{q}$, and $\mathbf{c}$ are computed solving the system equations defined by (3), and (4), with the $A$ matrix of (4) defined as

$$
A^{\prime}=\left(\begin{array}{ccc}
\frac{1}{a-\Delta d_{x}} & 0 & 0 \\
0 & \frac{1}{b-\Delta d_{y}} & 0 \\
0 & 0 & \frac{1}{c-\Delta d_{z}}
\end{array}\right)
$$

The complete expression for $\mathbf{c}$ is derived in [15], to which we refer for more details.

\section{B. Iterative Method}

The contact centroid $\mathbf{c}$, the scaling factor $K$ of the local torque $\mathbf{q}$, and the surface deformation $\Delta \mathbf{d}$ can be summarized into a vector $\mathbf{x}=\left[\mathbf{c}^{T}, K, \Delta \mathbf{d}^{T}\right]^{T}$. The identification of $\mathbf{x}$ can be achieved by solving the nonlinear system equations (7), that is derived from (1), (2), (5), and $\mathbf{f}=\mathbf{h}(\Delta \mathbf{d})$.

$$
g(\mathbf{x})=\left[\begin{array}{c}
K \frac{\partial S}{\partial c_{x}}-f_{y} c_{z}+f_{z} c_{y}-m_{x} \\
K \frac{\partial S}{\partial c_{y}}-f_{z} c_{x}+f_{x} c_{z}-m_{y} \\
K \frac{\partial S}{\partial c_{z}}-f_{y} c_{z}+f_{y} c_{x}-m_{z} \\
S^{\prime}(\mathbf{c}) \\
\mathbf{f}^{T} \mathbf{n}=h(\Delta \mathbf{d})^{T} \mathbf{n}
\end{array}\right]=0
$$

where $\mathbf{f}^{T} \mathbf{n}$ is the projection of $\mathbf{f}$ along the direction normal to $S^{\prime}$ in $\mathbf{c}$, and $\mathbf{h}(\Delta \mathbf{d})^{T} \mathbf{n}$ is the component of $\mathbf{h}(\Delta \mathbf{d})$ along the same direction.

The iterative method, we defined as (ii-a) in the Introduction, aims at solving $g(\mathbf{x})=0$, by using the LevenbergMarquardt (LM) method to minimize a positive defined cost function as in [19]. It has been shown that this method has a faster convergence time and better robustness, compared to other gradient-based methods [28].

The method needs an initial guess of the solution and if the initial guess is far from the ground truth, the required iterations could significantly increase or the algorithm even diverges. For this reason, a good initial guess is of paramount importance to improve the method performance. In this work, we tested the iterative method using a fix predefined position (i.e. the $\mathrm{CC}$ as would have happened on a rigid surface), referring to this case as to (ii-a); and the position calculated from the closed-form method (i) reported in Section III-A referred to as (ii-b). After the initial guess is defined, $g(\mathbf{x})=0$ is solved iteratively to get $\mathbf{x}$. Once the iterative algorithm satisfies the stopping criteria, the CC $\mathbf{c}$, the scaling factor $K$ of the local torque $\mathbf{q}$, and the surface deformation $\Delta \mathbf{d}$ are estimated.

\section{The Tactile Sensing Toolbox}

We provided a software implementation for methods (i), (iia), and (ii-b), developing the so-called Tactile Sensing Toolbox (TST). TST allows to acquire force and torque measurements and to retrieve contact centroid with the aforementioned techniques. The TST is implemented in $\mathrm{C}++$ under ROS on Ubuntu 14.04 installed PC (Intel $\AA$ ) Core ${ }^{\mathrm{TM}}$ i7-2600 CPU @ $3.40 \mathrm{GHz}, 16 \mathrm{~GB}$ RAM) with a loop rate of $200 \mathrm{~Hz}$. It is released under a 3-Clauses FreeBSD software license on GitHub and can be freely downloaded from https://github.com/ Kevin315/Pisa-Kings_Tactile_Sensing.git

In the following, we describe the functions of each node composing the TST:

- Sensor Node: it allows to set the force/torque (F/T) sensor parameters, e.g. data rate, units of measurement, cutoff frequency for low-pass filtering, and to perform sensor calibration. The force and torque values are published as a ROS message to be used by other nodes.

- Parameters Configuration: it provides a user-friendly interface to set the geometric parameters of the surface under exploration, e.g. surface principal axes $a, b$, and $c$ under the assumption of ellipsoidal geometries. It also enables to set the type of mathematical relation $\mathbf{h}(\cdot)$ between the applied force $\mathbf{f}$ and the surface deformation $\Delta \mathbf{d}$.

- ITS Problem Solving Node: this is the main node of the toolbox. It takes as input the published F/T data from the Sensor Node, and the parameters set through the Parameters Configuration routine. Then, this node computes the contact centroid on the specified surface using the received $\mathrm{F} / \mathrm{T}$ data, and selecting one of the methods (i), (ii-a) and (ii-b), reported in Section III-B The ITS problem result is published as a ROS message.

- ITS Problem Solution Visualization Node: it provides a visualization of the surface, the estimated contact centroid $\mathbf{c}$, resultant compressive force $\mathbf{p}$, and resultant moment $\mathbf{q}$ normal to the surface in the contact centroid.

- ITS Extra Info Node : this node provides the implementation of additional computational routines, which are not strictly needed for the $\mathbf{c}$ estimation, e.g. estimation of the linear and torsional friction coefficients relying on the theory in [6].

\section{EXPERIMENTAL SETUP FOR SPECIMENS CHARACTERIZATION AND VALIDATION}

The TST and the methods we presented in Section III were experimentally tested on silicone specimens. The specimens 
were first characterized in terms of force-indentation behavior, with the twofold aim of retrieving function $\mathbf{h}(\cdot)$ needed to implement our approach, and providing ground truth values for the validation of contact centroid estimation. To build silicones, room temperature curing silicone (BJB TC-5005 $\mathrm{A} / \mathrm{B})$ was mixed with a variable percentage of plasticizer (BJB TC-5005 C), acting as a softener. More specifically, two different percentages (over the total weight) of the amount of softener in the mixture were considered: $0 \%$ (specimen S0) and 20\% (specimen S20). The mixture was then poured in an ABS-printed mold to get ellipsoidal volume, with dimensions of $20 \times 20 \times 35 \mathrm{~mm}$. It is worth noticing that these specimens can be used to study the contact problem with soft surfaces under a twofold point of views: indeed, they not only represent the surface under exploration, but, in a complementary yet equivalent way, they can be regarded as a soft sensorized fingerpad interacting with a rigid indenter.

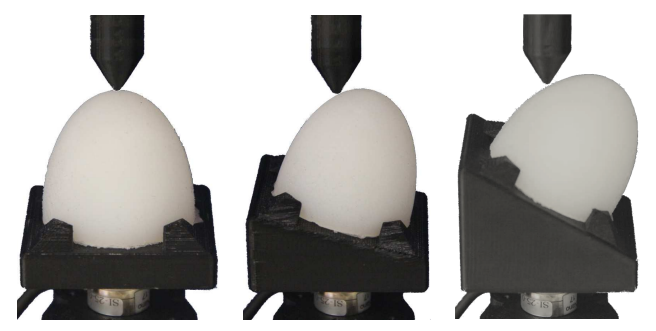

Fig. 3: Experimental setup. Both specimen were first characterized in the perpendicular configuration (leftmost), and then tested for two additional inclinations of the ellipsoid base with respect to the sensor plane.

A ProLine Z005 table-top testing machine by Zwick/Roell was used to characterize the mechanical properties of the specimens: as shown in Figure 3, the soft silicone ellipsoid was placed on an ABS support. For the characterization phase the support was fixed to the table. The indenter was built in ABS, with a conical shape with a total height of $30 \mathrm{~mm}$, and a base radius of $14 \mathrm{~mm}$, and a semi-spheric head of $2 \mathrm{~mm}$ radius. This ensures a localized contact with the object, providing reliable ground truth data for the estimation of $\mathrm{CC}$, as it was done in [16]. Indeed, the same indenter mounted on the testing machine was used also for the validation phase. In this case the ABS support was attached to an ATI Nano 17 Force/Torque sensor, with its $z_{s}$ axis aligned to the indenter main axis, leading to $\Delta d_{x}=\Delta d_{y}=0$ when the deformation is expressed in the sensor reference frame $\{S\}$. The force-indentation behavior was characterized at a speed of $5 \mathrm{~mm} / \mathrm{s}$, which is comparable to typical values observed in human indentation for softness discrimination [29]. During the validation phase, the ABS support was inclined, in order to get different tilt angles $\theta$ with respect to the vertical axis, as described later. These measurements were used to test the robustness of our method in providing reliable contact point estimation.

Figure 4 shows the force-indentation behavior that was obtained fro the specimen characterization. Two approximations were considered for fitting function $\mathbf{h}(\cdot):(a)$ a linear function, which comes with less accurate results but has the advantage
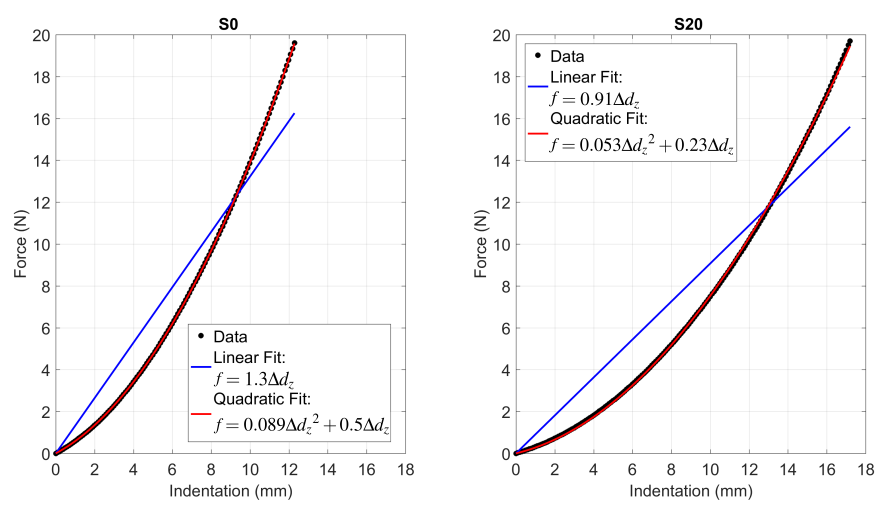

Fig. 4: Force-indentation characteristics.

to be simple to achieve; $(b)$ a quadratic function, which better models the characteristic of the material under investigation.

In the validation phase, the specimens were placed under the indenter with three different inclinations of the ellipsoid plane with respect to the table plane $(\theta=0,15$ and 30 degrees respectively), in order to evaluate performance on three different points over the ellipsoid surface. The indenter was controlled to press against the specimen with three different levels of force $(5,10$ and $15 \mathrm{~N})$, while coordinates for both measured and estimated $\mathrm{CC}$ were obtained in the ellipsoid reference frame $\{E\}$, using the proposed methods. The data acquisition loop rate for both surface characterization and validation phase was $50 \mathrm{~Hz}$. In the validation phase the applied force was held for 10 s, for each experimental value.

\section{RESUlTS}

Results were evaluated based on the following:

- Norm of the (vector) distance between the measured and estimated CC, both absolute and normalized with respect to the measured indentation;

- Angle between the estimated CC and the main ellipsoid axis $\left(z_{e}\right)$;

- For methods ii-a and ii-b, time of convergence of the algorithm for each single force measurement.

\begin{tabular}{|c|c|}
\hline Notation & Definition \\
\hline$\theta$ & Inclination (deg) \\
\hline$\|\mathbf{f}\|$ & Force $(\mathrm{N})$ \\
\hline$\Delta d_{z}$ & Indentation (mm) \\
\hline$\theta_{M}$ & $\begin{array}{l}\text { Angle between measured CC } \\
\text { and the } z_{e} \text { axis (deg) }\end{array}$ \\
\hline$e_{i}^{(\cdot)}, e_{i i_{a}}^{(\cdot)}, e_{i i_{b}}^{(\cdot)}$ & $\begin{array}{l}\text { Absolute errors on contact } \\
\text { points }(\mathrm{mm})\end{array}$ \\
\hline$e_{i \%}^{(\cdot)}, e_{i i_{a \%}}^{(\cdot)}, e_{i i_{b \%}}^{(\cdot)}$ & $\begin{array}{l}\text { Errors normalized by the in- } \\
\text { dentation }\end{array}$ \\
\hline$\theta_{i}^{(\cdot)}, \theta_{i i_{a}}^{(\cdot)}, \theta_{i i_{b}}^{(\cdot)}$ & $\begin{array}{l}\text { Angle between estimated CC } \\
\text { and the } z_{e} \text { axis (deg) }\end{array}$ \\
\hline$t_{i i_{a}}^{(\cdot)}, t_{i i_{b}}^{(\cdot)}$ & $\begin{array}{l}\text { Time to convergence for a sin- } \\
\text { gle force measurement }(\mathrm{ms})\end{array}$ \\
\hline$[\cdot]^{(l)},[\cdot]^{(q)}$ & $\begin{array}{l}\text { Results obtained using linear } \\
\text { and quadratic fitting }\end{array}$ \\
\hline$[\cdot]_{i},[\cdot]_{i i_{a}},[\cdot]_{i i_{b}}$, & $\begin{array}{l}\text { Results obtained using } \\
\text { method (i), (ii-a) and (ii-b) }\end{array}$ \\
\hline
\end{tabular}

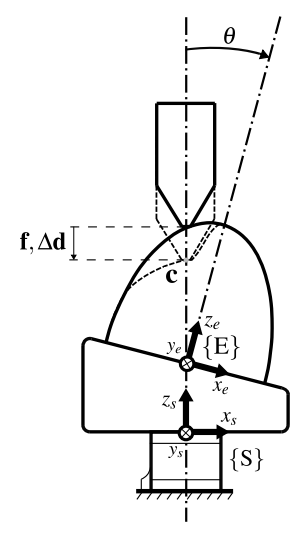

TABLE I: Notation for the validation analysis. 


\begin{tabular}{|c|c|c|c|c|c|c|c|c|c|c|c|c|c|c|c|c|c|}
\hline \multirow{3}{*}{$\theta$} & \multirow{3}{*}{$\|\mathbf{f}\|$} & \multicolumn{16}{|c|}{ Specimen } \\
\hline & & & \multicolumn{7}{|c|}{ So } & \multicolumn{8}{|c|}{ S20 } \\
\hline & & $\Delta d_{z}$ & $\theta_{M}$ & $e_{i}^{(l)}$ & $e_{i \%}^{(l)}$ & $\theta_{i}^{(l)}$ & $e_{i}^{(q)}$ & $e_{i \%}^{(q)}$ & $\theta_{i}^{(q)}$ & $\Delta d_{z}$ & $\theta_{M}$ & $e_{i}^{(l)}$ & $e_{i_{\%}}^{(l)}$ & $\theta_{i}^{(l)}$ & $e_{i}^{(q)}$ & $e_{i \%}^{(q)}$ & $\theta_{i}^{(q)}$ \\
\hline \multirow{3}{*}{0} & 5 & 5.54 & 1.02 & 0.74 & $13.44 \%$ & 0.83 & 0.30 & $5.50 \%$ & 0.82 & 7.77 & 1.10 & 1.20 & $15.49 \%$ & 0.89 & 0.16 & $2.07 \%$ & 0.90 \\
\hline & 10 & 8.42 & 1.13 & 1.12 & $13.24 \%$ & 0.78 & 0.27 & $3.26 \%$ & 0.81 & 11.87 & 1.30 & 1.24 & $10.45 \%$ & 0.93 & 0.22 & $1.84 \%$ & 0.91 \\
\hline & 15 & 10.60 & 1.23 & 3.66 & $34.47 \%$ & 0.75 & 0.20 & $1.92 \%$ & 0.82 & 14.90 & 1.50 & 4.71 & $31.57 \%$ & 1.11 & 0.26 & $1.71 \%$ & 0.95 \\
\hline \multirow{3}{*}{15} & 5 & 5.63 & 14.50 & 1.80 & $31.94 \%$ & 13.91 & 1.25 & $22.18 \%$ & 13.84 & 7.60 & 14.47 & 2.43 & $31.92 \%$ & 13.13 & 1.17 & $15.40 \%$ & 12.92 \\
\hline & 10 & 8.51 & 14.45 & 0.94 & $11.06 \%$ & 13.14 & 1.62 & $19.08 \%$ & 13.22 & 11.59 & 14.37 & 1.33 & $11.45 \%$ & 11.78 & 1.75 & $15.12 \%$ & 11.89 \\
\hline & 15 & 10.69 & 14.39 & 1.64 & $15.37 \%$ & 12.32 & 1.82 & $17.01 \%$ & 12.64 & 14.54 & 14.27 & 2.82 & $19.40 \%$ & 10.41 & 1.97 & $13.51 \%$ & 11.09 \\
\hline \multirow{3}{*}{30} & 5 & 5.70 & 27.89 & 2.27 & $39.74 \%$ & 27.79 & 1.48 & $25.94 \%$ & 27.57 & 7.40 & 27.72 & 3.05 & $41.19 \%$ & 26.59 & 1.36 & $18.43 \%$ & 26.10 \\
\hline & 10 & 8.51 & 27.60 & 1.98 & $23.22 \%$ & 26.36 & 2.02 & $23.70 \%$ & 26.37 & 11.26 & 27.24 & 2.66 & $23.66 \%$ & 24.60 & 2.12 & $18.86 \%$ & 24.38 \\
\hline & 15 & 10.55 & 27.34 & 1.16 & $11.04 \%$ & 24.74 & 2.26 & $21.42 \%$ & 25.33 & 14.24 & 26.70 & 1.50 & $10.52 \%$ & 22.69 & 2.56 & $17.97 \%$ & 23.30 \\
\hline \multirow{2}{*}{\multicolumn{4}{|c|}{$\begin{array}{l}\text { Averages } \\
\text { Std. deviation }\end{array}$}} & 1.70 & $21.50 \%$ & & 1.25 & $15.56 \%$ & & & & 2.33 & $21.74 \%$ & & 1.29 & $11.66 \%$ & \\
\hline & & & & \pm 0.89 & \pm 11.19 & & \pm 0.80 & \pm 9.39 & & & & \pm 1.16 & \pm 11.10 & & \pm 0.90 & \pm 7.54 & \\
\hline
\end{tabular}

TABLE II: Summary of CC estimation accuracy using method (i).

\begin{tabular}{|c|c|c|c|c|c|c|c|c|c|c|c|c|c|c|c|c|c|c|c|c|}
\hline \multirow{3}{*}{$\theta$} & \multirow{3}{*}{$\|\mathbf{f}\|$} & \multicolumn{19}{|c|}{ Specimen } \\
\hline & & \multicolumn{10}{|c|}{ S0 } & \multicolumn{9}{|c|}{ S20 } \\
\hline & & $\Delta d_{z}$ & $\theta_{M}$ & $e_{i i_{a}}^{(l)}$ & $e_{i a_{a \%}}^{(l)}$ & $\theta_{i i_{a}}^{(l)}$ & $t_{i i_{a}}^{(l)}$ & $e_{i i_{a}}^{(q)}$ & $e_{i i_{a \%}}^{(q)}$ & $\theta_{i i_{a}}^{(q)}$ & $t_{i i_{a}}^{(q)}$ & $\Delta d_{z}$ & $\theta_{M}$ & $e_{i i_{a}}^{(l)}$ & $e_{i i_{a \%}}^{(l)}$ & $\theta_{i i_{a}}^{(l)}$ & $t_{i i_{a}}^{(l)}$ & $e_{i i_{a}}^{(q)} e_{i i_{a \%}}^{(q)}$ & $\theta_{i i_{a}}^{(q)}$ & $t_{i i_{a}}^{(q)}$ \\
\hline \multirow{3}{*}{0} & 5 & 5.54 & 1.02 & 1.65 & $29.98 \%$ & 0.44 & 0.81 & N/A & N/A & N/A & N/A & 7.77 & 1.10 & 2.38 & $30.54 \%$ & 0.31 & 0.67 & N/A N/A & N/A & N/A \\
\hline & 10 & 8.42 & 1.13 & 0.77 & $9.18 \%$ & 0.44 & 0.59 & N/A & N/A & N/A & N/A & 11.87 & 1.30 & 1.24 & $10.33 \%$ & 0.30 & 0.57 & N/A N/A & N/A & N/A \\
\hline & 15 & 10.60 & 1.23 & 0.40 & $3.59 \%$ & 0.41 & 1.21 & N/A & N/A & N/A & N/A & 14.90 & 1.50 & 1.04 & $6.91 \%$ & 0.29 & 0.19 & N/A N/A & N/A & N/A \\
\hline \multirow{3}{*}{15} & 5 & 5.63 & 14.50 & 1.92 & $34.29 \%$ & 13.91 & 0.34 & N/A & N/A & N/A & N/A & 7.60 & 14.47 & 2.45 & $32.21 \%$ & 13.15 & 0.56 & N/A N/A & N/A & N/A \\
\hline & 10 & 8.51 & 14.45 & 1.51 & $17.71 \%$ & 13.16 & 0.47 & N/A & N/A & N/A & N/A & 11.59 & 14.37 & 1.99 & $16.56 \%$ & 12.84 & 0.31 & N/A N/A & N/A & N/A \\
\hline & 15 & 10.69 & 14.39 & 0.38 & $3.44 \%$ & 12.35 & 0.35 & N/A & N/A & N/A & N/A & 14.54 & 14.27 & 0.18 & $1.18 \%$ & 12.02 & 0.20 & N/A N/A & N/A & N/A \\
\hline \multirow{3}{*}{30} & 5 & 5.70 & 27.89 & 2.03 & $35.55 \%$ & 27.66 & 0.18 & N/A & N/A & N/A & N/A & 7.40 & 27.72 & 2.32 & $31.33 \%$ & 26.39 & 0.17 & N/A N/A & N/A & N/A \\
\hline & 10 & 8.51 & 27.60 & 1.29 & $15.14 \%$ & 26.09 & 0.80 & N/A & N/A & N/A & N/A & 11.26 & 27.24 & 1.03 & $9.40 \%$ & 24.13 & 0.19 & N/A N/A & N/A & N/A \\
\hline & 15 & 10.55 & 27.34 & 0.68 & $6.17 \%$ & 24.27 & 0.46 & N/A & N/A & N/A & N/A & 14.24 & 26.70 & 0.38 & $2.73 \%$ & 25.62 & 0.47 & N/A N/A & N/A & N/A \\
\hline \multirow{2}{*}{\multicolumn{4}{|c|}{$\begin{array}{l}\text { Averages } \\
\text { Std. deviation }\end{array}$}} & 1.18 & $17.23 \%$ & & 0.58 & N/A & N/A & N/A & N/A & & & 1.47 & $15.69 \%$ & & 0.37 & N/A N/A & N/A & N/A \\
\hline & & & & \pm 0.64 & $\pm 13.03 \%$ & & \pm 0.31 & N/A & N/A & N/A & N/A & & & \pm 0.84 & $\pm 12.56 \%$ & & \pm 0.20 & N/A N/A & N/A & N/A \\
\hline
\end{tabular}

TABLE III: Summary of CC estimation accuracy using method (ii-a).

\begin{tabular}{|c|c|c|c|c|c|c|c|c|c|c|c|c|c|c|c|c|c|c|c|c|c|}
\hline \multirow{3}{*}{$\theta$} & \multirow{3}{*}{$\|\mathbf{f}\|$} & \multicolumn{20}{|c|}{ Specimen } \\
\hline & & \multicolumn{10}{|c|}{ So } & \multicolumn{10}{|c|}{ S20 } \\
\hline & & $\Delta d_{z}$ & $\theta_{M}$ & $e_{i i_{b}}^{(l)}$ & $e_{i b_{b \%}}^{(l)}$ & $\theta_{i i_{b}}^{(l)}$ & $t_{i i_{b}}^{(l)}$ & $e_{i i_{b}}^{(q)}$ & $e_{i i_{b \%}}^{(q)}$ & $\theta_{i i_{b}}^{(q)}$ & $t_{i i_{b}}^{(q)}$ & $\Delta d_{z}$ & $\theta_{M}$ & $e_{i i_{b}}^{(l)}$ & $e_{i b_{b \%}}^{(l)}$ & $\theta_{i i_{b}}^{(l)}$ & $t_{i i_{b}}^{(l)}$ & $e_{i i_{b}}^{(q)}$ & $e_{i i b_{\%}}^{(q)}$ & $\theta_{i i_{b}}^{(q)}$ & $t_{i i_{b}}^{(q)}$ \\
\hline \multirow{3}{*}{0} & 5 & 5.54 & 1.02 & 1.65 & $29.98 \%$ & 0.45 & 0.19 & 0.42 & $7.68 \%$ & 0.45 & 0.42 & 7.77 & 1.10 & 2.39 & $30.61 \%$ & 0.31 & 0.20 & 0.23 & $3.00 \%$ & 0.30 & 0.29 \\
\hline & 10 & 8.42 & 1.13 & 0.77 & $9.19 \%$ & 0.44 & 0.13 & 0.16 & $1.84 \%$ & 0.45 & 0.60 & 11.87 & 1.30 & 1.19 & $9.91 \%$ & 0.30 & 0.14 & 0.03 & $0.24 \%$ & 0.29 & 0.33 \\
\hline & 15 & 10.60 & 1.23 & 0.40 & $3.61 \%$ & 0.42 & 0.12 & 0.59 & $5.53 \%$ & 0.42 & 0.40 & 14.90 & 1.50 & 1.10 & $7.30 \%$ & 0.30 & 0.14 & 0.75 & $5.02 \%$ & 0.27 & 0.30 \\
\hline \multirow{3}{*}{15} & 5 & 5.63 & 14.50 & 1.92 & $34.32 \%$ & 13.91 & 0.17 & 0.09 & $1.68 \%$ & 14.44 & 0.56 & 7.60 & 14.47 & 2.44 & $32.17 \%$ & 13.15 & 0.13 & 0.00 & $0.04 \%$ & 13.93 & 0.54 \\
\hline & 10 & 8.51 & 14.45 & 1.49 & $17.55 \%$ & 13.17 & 0.18 & 0.42 & $4.92 \%$ & 13.86 & 0.70 & 11.59 & 14.37 & 1.99 & $16.60 \%$ & 13.02 & 0.13 & 0.65 & $5.57 \%$ & 13.12 & 0.40 \\
\hline & 15 & 10.69 & 14.39 & 0.52 & $4.77 \%$ & 12.35 & 0.14 & 0.95 & $8.85 \%$ & 13.43 & 0.34 & 14.54 & 14.27 & 0.27 & $1.80 \%$ & 12.29 & 0.13 & 1.20 & $8.22 \%$ & 12.55 & 0.82 \\
\hline \multirow{3}{*}{30} & 5 & 5.70 & 27.89 & 2.03 & 3. & 27.67 & 0. & 0. & $2.71 \%$ & 29.59 & 0.3 & 7.40 & 27.72 & 2.32 & 3 & 26.40 & 0.13 & 0.20 & 2. & 28.39 & 0.46 \\
\hline & 10 & 8.51 & 27.60 & 1.30 & $15.26 \%$ & 26.10 & 0.55 & 0.38 & $4.47 \%$ & 28.98 & 0.80 & 11.26 & 27.24 & 1.17 & $10.64 \%$ & 24.13 & 0.13 & 0.72 & $6.41 \%$ & 27.50 & 0.25 \\
\hline & 15 & 10.55 & 27.34 & 0.51 & $4.62 \%$ & 24.28 & 0.13 & 0.34 & $3.21 \%$ & 28.62 & 0.36 & 14.24 & 26.70 & 0.40 & $2.89 \%$ & 25.62 & 0.17 & 1.09 & $7.64 \%$ & 26.83 & 0.37 \\
\hline & & & & 1.18 & $17.21 \%$ & & 0.19 & 0.39 & $4.54 \%$ & & 0.50 & & & 1.47 & $15.92 \%$ & & 0.15 & 0.54 & $4.32 \%$ & & 42 \\
\hline & dev & & & \pm 0.64 & \pm 13.04 & & \pm 0.13 & \pm 0.26 & \pm 2.50 & & \pm 0.17 & & & \pm 0.84 & \pm 12.37 & & \pm 0.02 & \pm 0.44 & \pm 3.00 & & \pm 0.17 \\
\hline
\end{tabular}

TABLE IV: Summary of CC estimation accuracy using method (ii-b). 
Table I describes the notations used for each of these quantities, while tables II to IV show details of the estimation results. Results are reported in terms of average values over the 10 s holding phase. It is worth pointing out that the maximum standard deviation of the observed force/torque measurements was under $0.02 \mathrm{~N}$ and $0.06 \mathrm{~N}$ mm, respectively. Table $[\mathrm{II}]$ shows results using method (i), i.e. direct closed form calculation on a shrunk ellipsoid. As expected, results obtained with the linear approximation yield a higher error on the $\mathrm{CC}$ estimation, with a peak error of around $41 \%$ of the indentation for the S20 specimen. Considering that the standard ITS approach would yield an error of $100 \%$, and that the absolute value of the position estimation error never exceeded $5 \mathrm{~mm}$, this could still be acceptable, at least for some applications. However, using the quadratic approximation lead to a more reliable performance, with a maximum absolute error below $3 \mathrm{~mm}$ and the relative error never exceeding $26 \%$ of the indentation. The maximum errors on the estimation of the inclination of $\mathrm{CC}$ with respect to the main ellipsoid axis are about 4 degrees for both approximations.

Table III shows results obtained with method (ii-a), where the iterative approach presented in [19] is applied by using the rigid (initial) contact as initial guess. With this method it was only possible to obtain results with linear approximation; indeed, the algorithm failed to converge when using the quadratic approximation of the force-indentation behavior, which was also reported as an issue with this method in [19]. Results for the linear approximation show lower errors than those obtained with method (i) when also using a linear approximation, but slightly higher when compared with method (i) and quadratic approximation, peaking at around $2.5 \mathrm{~mm}$ and $35 \%$ of the indentation, with the maximum error on the estimated inclination of the point being about 3 degrees. The maximum value of the time to convergence of the algorithm was $1.21 \mathrm{~ms}$, which would enable a frequency cycle above $800 \mathrm{~Hz}$, well above the $200 \mathrm{~Hz}$ limit imposed by ROS.

The best result is obtained when combining the two approaches, i.e. by using the iterative method with the outcome from (i) as initial guess, as shown in Table [V] For what concerns results obtained with the linear approximation, errors seems to be comparable with those obtained with method (ii-a), but the time to convergence was lowered for every point considered. The most important results however is that the algorithm is now able to converge even when using the quadratic approximation. This leads to errors on the estimated $\mathrm{CC}$ that are of the order of the $1 \mathrm{~mm}$ and always below $9 \%$ of the indentation. Convergence times, while being higher than those obtained with method (ii-b) if using the linear approximation, are always below $1 \mathrm{~ms}$, and thus still suitable for real-time applications.

\section{CONCLUSIONS AND FUTURE WORKS}

In this paper, we described an approach to estimate location of contacts on soft surfaces, when a characterization of the soft material in terms of force-indentation is available. We presented two methods: (i) a closed form solution, where the location of contacts is estimated using standard ITS on a shrunk ellipsoidal surface, for which the geometry is obtained from the characterization, and (ii) the iterative method introduced in [19], which casts the problem of finding the contact on a soft surface, as an iterative optimization problem. This method is applied by using as initial guess (ii-a) the rigid $\mathrm{CC}$, and (ii-b) the results from method (i). These techniques were implemented in a ROS-based toolbox, the Tactile Sensing Toolbox, and experimentally tested on two silicone specimens. These specimens were built to have different levels of stiffness, and characterized, considering linear and quadratic approximations for the force-indentation behavior. The quality of $\mathrm{CC}$ estimation was evaluated by comparing the outcomes with the measurements obtained with the testing machine.

Results show that the accuracy is limited when using a linear approximation: direct calculation with method (i) yields errors that peak at $5 \mathrm{~mm}$ and $41 \%$ of the indentation, while methods (ii-a) and (ii-b) improve the accuracy bringing maximum errors to $2.5 \mathrm{~mm}$ and $35 \%$ of the indentation. Where the approach really shines however is when using the more accurate quadratic interpolation of the force-indentation behavior. This already improves the performance for method (i), with a maximum error of $3 \mathrm{~mm}$ and $26 \%$ of the indentation; for what concerns the iterative method, it was not possible to obtain convergence of the algorithm when using the initial contact as initial guess with method (ii-a), while method (ii-b) yields maximum errors of the order of $1 \mathrm{~mm}$ and less than $9 \%$ of the indentation. Using the outcome of method (i) as initial guess for (ii) proves in general to be able to reduce convergence times, which stay lower than $1 \mathrm{~ms}$ even when using the quadratic interpolation. These results open promising perspectives for applications to robotic grasp control and/or remote environment tele-exploration through soft sensors or fingerpads.

Conclusions that can be drawn are that, when a good characterization of the material is available, it is possible to estimate the location of the contact centroid with good accuracy also for deformable materials. In this paper the best results were obtained when approximating the forceindentation behavior with a quadratic function; however, it is reasonable to believe that good results would have been obtained with a linear approximation if the deformable specimens had a linear behavior. Future works will be devoted to test our methods with a larger set of materials and experimental conditions, e.g. velocity, geometry, and to evaluate extensions of our approach to the case of soft vs. soft surface contact. Finally, an implementation of these techniques contained in the ROS-based toolbox to develop a sensorized soft fingerpads as in [19] is also envisioned.

\section{ACKNOWLEDGMENTS}

This work is supported in part by the EU H2020 projects SoftPro (No. 688857) and SOMA (No. 645599), and by the EPSRC UK in the ESSENCE Project (Grant No: EP/N020421/1).

\section{REFERENCES}

[1] A. Bicchi, "On the closure properties of robotic grasping," The International Journal of Robotics Research, vol. 14, no. 4, pp. 319-334, 1995. 
[2] B. Siciliano and O. Khatib, Springer handbook of robotics. Springer, 2016.

[3] W.-C. Choi, "Polymer micromachined flexible tactile sensor for threeaxial loads detection," Transactions on electrical and electronic materials, vol. 11, no. 3, pp. 130-133, 2010.

[4] B. D. Argall and A. G. Billard, "A survey of tactile human-robot interactions," Robotics and Autonomous Systems, vol. 58, no. 10, pp. 1159 - 1176, 2010. [Online]. Available: http://www.sciencedirect.com/ science/article/pii/S0921889010001375

[5] D. Silvera-Tawil, D. Rye, and M. Velonaki, "Artificial skin and tactile sensing for socially interactive robots: A review," Robotics and Autonomous Systems, vol. 63, pp. 230-243, 2015.

[6] C. Melchiorri, "Slip detection and control using tactile and force sensors," Mechatronics, IEEE/ASME Transactions on, vol. 5, no. 3, pp. 235-243, Sep 2000.

[7] J. S. Son and R. D. Nowe, "Tactile sensing and stiffness control with multifingered hands," in Proceedings of IEEE International Conference on Robotics and Automation, vol. 4, Apr 1996, pp. 3228-3233 vol.4.

[8] W. Chen, H. Khamis, I. Birznieks, N. F. Lepora, and S. J. Redmond, "Tactile sensors for friction estimation and incipient slip detection - towards dexterous robotic manipulation: A review," IEEE Sensors Journal, pp. 1-1, 2018.

[9] D. Prattichizzo and J. C. Trinkle, Chapter "Grasping" in the Springer Handbook of Robotics. Springer, 2016.

[10] J. Engel, J. Chen, and C. Liu, "Development of polyimide flexible tactile sensor skin," Journal of Micromechanics and Microengineering, vol. 13, no. 3, p. 359, 2003.

[11] G. Cannata, M. Maggiali, G. Metta, and G. Sandini, "An embedded artificial skin for humanoid robots," in Multisensor Fusion and Integration for Intelligent Systems, 2008. MFI 2008. IEEE International Conference on. IEEE, 2008, pp. 434-438.

[12] R. D. P. Wong, J. D. Posner, and V. J. Santos, "Flexible microfluidic normal force sensor skin for tactile feedback," Sensors and Actuators A: Physical, vol. 179, pp. 62-69, 2012.

[13] M. W. Strohmayr and D. Schneider, "The dlr artificial skin step ii: Scalability as a prerequisite for whole-body covers," in 2013 IEEE/RSJ International Conference on Intelligent Robots and Systems, Nov 2013, pp. $4721-4728$.

[14] G. H. Büscher, R. Kõiva, C. Schürmann, R. Haschke, and H. J. Ritter "Flexible and stretchable fabric-based tactile sensor," Robotics and Autonomous Systems, vol. 63, pp. 244-252, 2015.

[15] A. Bicchi, J. K. Salisbury, and D. L. Brock, "Contact sensing from force and torque measurements," The Int. J. of Robotics Research, vol. 12, no. 3, pp. 249-262, 1993 .

[16] E. Battaglia, G. Grioli, M. G. Catalano, M. Santello, and A. Bicchi, "Thimblesense: An individual-digit wearable tactile sensor for experimental grasp studies," in 2014 IEEE International Conference on Robotics and Automation (ICRA), May 2014, pp. 2728-2735.

[17] A. Altobelli, M. Bianchi, M. G. Catalano, A. Serio, G. Baud-Bovy, and A. Bicchi, "An instrumented manipulandum for human grasping studies," in 2015 IEEE International Conference on Rehabilitation Robotics (ICORR), Aug 2015, pp. 169-174.

[18] A. Serio, E. Riccomini, V. Tartaglia, I. Sarakoglou, M. Gabiccini, N. Tsagarakis, and A. Bicchi, "The patched intrinsic tactile object: A tool to investigate human grasps," in 2014 IEEE/RSJ International Conference on Intelligent Robots and Systems, Sept 2014, pp. 12611268.

[19] H. Liu, K. C. Nguyen, V. Perdereau, J. Bimbo, J. Back, M. Godden, L. D. Seneviratne, and K. Althoefer, "Finger contact sensing and the application in dexterous hand manipulation," Autonomous Robots, vol. 39, no. 1, pp. 25-41, 2015.

[20] J. Back, J. Bimbo, Y. Noh, L. D. Seneviratne, K. Althoefer, and H. Liu, "Control a contact sensing finger for surface haptic exploration." in ICRA, 2014, pp. 2736-2741.

[21] T. Sun, J. Back, and H. Liu, "Combining contact forces and geometry to recognize objects during surface haptic exploration," IEEE Robotics and Automation Letters, vol. 3, no. 3, pp. 2509-2514, 2018.

[22] Z. Su, K. Hausman, Y. Chebotar, A. Molchanov, G. E. Loeb, G. S. Sukhatme, and S. Schaal, "Force estimation and slip detection/classification for grip control using a biomimetic tactile sensor," in Humanoid Robots (Humanoids), 2015 IEEE-RAS 15th International Conference on. IEEE, 2015, pp. 297-303.

[23] B. Ward-Cherrier, N. Pestell, L. Cramphorn, B. Winstone, M. E. Giannaccini, J. Rossiter, and N. F. Lepora, "The tactip family: Soft optical tactile sensors with 3d-printed biomimetic morphologies," Soft
Robotics, vol. 5, no. 2, pp. 216-227, 2018, pMID: 29297773. [Online]. Available: https://doi.org/10.1089/soro.2017.0052

[24] E. Miguel, M. L. D’Angelo, F. Cannella, M. Bianchi, M. Memeo, A. Bicchi, D. G. Caldwell, and M. A. Otaduy, "Characterization of nonlinear finger pad mechanics for tactile rendering," in World Haptics Conference (WHC), 2015 IEEE. IEEE, 2015, pp. 63-68.

[25] M. Ciocarlie, A. Miller, and P. Allen, "Grasp analysis using deformable fingers," in 2005 IEEE/RSJ International Conference on Intelligent Robots and Systems, Aug 2005, pp. 4122-4128.

[26] T. Tsuji, Tactile Sensing Techniques That Use Intrinsic Force Sensors. Tokyo: Springer Japan, 2016, pp. 139-155.

[27] K. L. Johnson, Contact Mechanics. Cambridge University Press, 1985.

[28] Y. Bard, "Comparison of gradient methods for the solution of nonlinear parameter estimation problems," SIAM Journal on Numerical Analysis, vol. 7, no. 1, pp. 157-186, 1970 .

[29] M. A. Srinivasan and R. H. LaMotte, "Tactual discrimination of softness," Journal of Neurophysiology, vol. 73, no. 1, pp. 88-101, 1995. 